\title{
Aeroelastic simulations of a delta wing with a Chimera approach for deflected control surfaces
}

\author{
Christopher Reinbold $^{1}\left[\right.$ D $\cdot$ Kaare Sørensen ${ }^{2} \cdot$ Christian Breitsamter $^{1}$
}

Received: 22 March 2021 / Revised: 3 September 2021 / Accepted: 11 October 2021 / Published online: 12 November 2021

(c) The Author(s) 2021

\begin{abstract}
A numerical tool for the computation of aircraft control surface aerodynamics with flexibility effects is presented. The solution is based on coupled Computational Fluid Dynamics (CFD) and Computational Structural Mechanics (CSM) simulations embedded in the multidisciplinary simulation environment SimServer. In SimServer, the DLR-TAU Code is utilized to obtain the CFD solution by solving the Reynolds-Averaged Navier-Stokes (RANS) equations. Structural displacements are computed with a modal solver. The Chimera implementation of SimServer, suited for hybrid grids, is applied to model the control surfaces. Numerical simulations with the flexible Chimera method are performed for the Model53 wing configuration, which is a generic delta wing with a deployed slat as well as an inboard and outboard trailing edge flap. Aerodynamic and aeroelastic simulations at high dynamic pressure $q=45 \mathrm{kPa}$ and transonic speed $\mathrm{Ma}=0.8$ are performed for several angles of attack $10^{\circ} \leq \alpha \leq 25^{\circ}$ and flap deflection angles $-30^{\circ} \leq \delta \leq 30^{\circ}$. The effect of structural deformations on the flow field and control surface effectiveness are analyzed and compared to computations of components treated fully rigid. At the targeted freestream condition $M=0.8$ and Re $=15.1 \times 10^{7}$, the flow field around the Model53 configuration is characterized by the interaction of vortices and shock waves. The results of the lift and pitching moment coefficient for the rigid and flexible configuration revealed the importance of taking the structural flexibility into account in order to obtain more accurate results for the considered range of flap deflections. Furthermore, the computational effort of the aerodynamic and aeroelastic simulations are evaluated. The increase in computational effort is shown to be adequate for the given increase in accuracy.
\end{abstract}

Keywords Aeroelasticity $\cdot$ Delta wing $\cdot$ Control surfaces $\cdot$ Chimera

\section{Introduction}

The influence of control surface deflections on the aerodynamic characteristics of an aircraft is of high interest in aerospace engineering. In particular, highly loaded flaps, e.g. for high agility aircraft [1-3], may cause large structural deformations, thus degrading the aerodynamic efficiency due to changes in spanwise flap gap adjustments, flap positions and wing twist. Aircraft maneuvers or trim state simulations with Computational Fluid Dynamics (CFD) solvers require the treatment of control surface deflections [4]. The

Christopher Reinbold

christopher.reinbold@tum.de

1 Chair of Aerodynamics and Fluid Mechanics, Technical University of Munich, Boltzmannstr. 15, 85748 Garching, Germany

2 Flight Physics - Aerodynamics, Airbus Defence \& Space, Manching, Germany movement of control surfaces in high-fidelity simulations is a challenging task. Re-meshing for each deflection angle is computationally expensive and not trivial for time-dependent motions [5]. Mesh deformation methods are problematic in combination with large deployments and do not allow for the opening of gaps between control surfaces and the wing. The Chimera technique, however, offers the possibility to model the deployment of control surfaces realistically, while enabling movements during a simulation without the necessity of re-meshing the computational grid. Thus, the efficiency of computing a series of deflection angles or an aerodynamic dataset for an aircraft configuration can be significantly improved with the Chimera method. To further increase the accuracy of such computations, structural stiffness needs to be considered due to their effect on control surface efficiency and the aerodynamic characteristics of the aircraft in general.

Over the last years, the modeling of control surface deflections with CFD solvers has been subject to several 
projects, such as ComFlite [6], Digital-X [7], VicToria and Mephisto [8], as well as several publications, for instance by Vos et al. [9], Sørensen et al. [10] and Clifton et al. [11]. Similarly, aeroelastic simulations of aircraft using coupled iterative CFD-CSM simulations with high-fidelity CFD methods (RANS or higher precision) and detailed structural models have been investigated by several authors such as Voß et al. [12], Fornasier et al. [13] and Stickan et al. [14]. However, the combination of coupled high-fidelity CFD-CSM simulations with an accurate and efficient modeling of control surfaces is still an ongoing research topic. The focus of this work is the introduction and application of a numerical method based on the multidisciplinary simulation environment SimServer [15], which is capable of conducting coupled CFD-CSM simulation with control surface deflections. The modeling of control surfaces is performed with a Chimera approach for hybrid structured/unstructured grids, which ensures a robust, accurate and efficient handling of control surface deflections for aircraft configurations up to industrial complexity [10]. Due to the compatibility of the Chimera implementation with hybrid grids as well as intersecting surfaces, it offers the possibility to handle complex geometries as well as different types of control surfaces. Hence, the presented solution is considered to be advantageous compared to methods which are using sliding mesh approaches as described in [5], since the Chimera technique is more robust combined with mesh deformation, or to solutions which are limited to structured grids only, as presented in [9], where the mesh generation process can be time-consuming and difficult for complex geometries.

First, the numerical methods which are involved in the aeroelastic simulations are described. Subsequently, the Model53 wing geometry as well as the aerodynamic and structural grid is presented. Finally, the results obtained with the flexible Chimera method are analyzed for the Model53 at transonic speed and high dynamic pressure.

\section{Numerical methods}

This chapter describes the applied numerical methods. The CFD-solver, the fluid-structure coupling and the CFD-grid deformation is introduced. Thereafter, the CSM-solver, the Chimera implementation and the CFD-CSM coupling process are described. All methods are implemented into the SimServer multidisciplinary simulation environment [15]. Emphasis is put on the integration of the Chimera method into the partitioned approach of SimServer for solving aeroelastic problems.

\subsection{Computational fluid dynamics solver}

The DLR-TAU flow code is employed in the SimServer environment to solve the compressible, three-dimensional Reynolds-Averaged Navier Stokes (RANS) equations [16]. It uses a vertex-centered dual cell method for hybrid, unstructured grids. Even though steady-state cases are targeted, the aerodynamic and aeroelastic simulations are performed as URANS (Unsteady-RANS) computations with a dual-time stepping and an implicit Backward-Euler scheme. A time step of $\Delta t=0.01 \mathrm{~s}$ is used, which corresponds to a characteristic time step of $\Delta t^{*}=\left(\Delta t U_{\infty} / l_{\mu}\right)=0.5$, where $U_{\infty}$ is the freestream velocity and $l_{\mu}$ the mean aerodynamic chord. A comparison of CFD simulations with different time steps $0.001 \mathrm{~s}<\Delta t<0.05 \mathrm{~s}$ and a steady-state RANS simulation revealed no difference with regard to the final results. The time step of $\Delta t=0.01 \mathrm{~s}$ is chosen, as it provided stable and fast convergence. The inner iterations are set to $i=50$ but can be reduced to $i=25$ by the solver if the convergence criterion is achieved for the lift and pitching moment coefficients, which is $\left|C_{\mathrm{L}}\right|<0.005$ and $\left|C_{\mathrm{My}}\right|<0.001$ within the last 500 iterations. After reaching the convergence criterion, the specified time steps for each deflection angle are completed but continued with the reduced inner iterations, so that a reduction in computational effort can be achieved. The inner iterations are reset to $i=50$ after each flap rotation. In addition, convergence acceleration is achieved with the multigrid technique, for which a $3 \mathrm{w}$ cycle is utilized, using point-implicit relaxation. For the spatial discretization, a finite-volume formulation with second-order upwind scheme is applied. The RANS equations are closed with the Spalart-Allmaras one-equation turbulence model [17] in the Edward's modified version with rotational correction [18].

\subsection{Fluid-structure coupling}

For the computation of weakly coupled aeroelastic simulations with movable control surfaces, an important requirement to the fluid-structure coupling is the compatibility with the Chimera implementation. The existing coupling algorithms in SimServer did not fulfill this requirement, especially as problems arose in combination with intersecting geometries, that can occur in combination with Chimera. Thus, a new coupling algorithm has been developed for transferring forces and displacements between the surface nodes of the CFD-grid and the surface nodes of the structural finite-element (FE) model. The interpolation method is based on the point-element-relationship between the CFD- and the CSM-surface grid and has been 
validated with the BACT [19] and HIRENASD [20] test case. The point-element relationships are computed by a nearest neighbour search, which is based on finding the nearest CSM element for each CFD node.

The point-element-relationships are initialized with the CFD and CSM grid in their undeflected flap configuration during the first coupling exchange cycle. To reduce the computational effort for subsequent coupling cycles, the point-element-relationships are stored in registers. For the transfer of forces and displacements, the deflection of control surfaces is only considered for the CFD grid, while the CSM flaps are undeflected. The forces are evaluated on the deflected flaps of the CFD grid and then transferred to the undeflected CSM grid by maintaining the direction of the force vectors, see Fig. 1. Similarly, the displacements are computed on the undeflected FE model and coupled to the deflected CFD grid by maintaining the direction of the displacement vectors.

Compatibility with the Chimera implementation of SimServer is ensured by allowing the user to define the surface IDs of the structural and aerodynamic grid where data should be exchanged. Hence, the transfer of forces and displacements in Chimera regions, e.g. flaps, can be performed independently of other regions, such as the main wing or the fuselage.

Another important aspect in the case where the rotation of flaps are targeted in aeroelastic simulations is the adjustment of the hinge line axis according to the deformations of the wing. To take this into account, the coordinates of the two points defining the hinge axis of each flap are displaced by employing the same algorithm used for the transfer of displacements from the CSM to the CFD grid.

\subsection{CFD volume grid deformation}

The surface displacements are propagated to the CFD volume grid with a parallel mesh deformation method based on an advancing front algorithm. Since the repair of badly distorted elements was not available in the original code, a local re-meshing procedure according to Ref. [21] has been implemented in order to support larger local deformations. The repair procedure marks regions in the grid containing collapsed elements surrounded by valid cells only. Subsequently, the collapsed cells are rebuilt by employing a volume spline interpolation approach. More information on the mesh deformation method can be found in Refs. [15, 21].

\subsection{Chimera implementation}

The Chimera technique is an efficient way to compute multiple body problems, such as control surface deflections, for hybrid grids [22]. In general, it assembles two or more grids into one computational domain through the application of interpolation boundary conditions. To apply the Chimera method in SimServer, one or more submeshes need to be contained within a background mesh. In case of a wing configuration with control surfaces, the wing mesh corresponds to the background mesh, whereas each control surface mesh corresponds to a submesh. Geometries belonging to the background mesh or submesh are allowed to intersect. A detailed explanation of the interpolation method and the hole-cutting procedure can be found in Ref. [10].

\subsection{Structural solver}

The structural deformations for the static aeroelastic simulations in this work are computed with a linear modal solver. The linear approach is justifiable for the investigated configuration, since the overall elastic deformations remain small with a wing tip deformation of approx. $4 \%$ relative to the semi-span. A mass-normalized modal matrix $\boldsymbol{\Phi}_{\mathrm{s}}$, which contains the structural eigenmodes obtained by an eigenvalue analysis, is used to write the physical structural displacements in modal coordinates $\mathbf{q}$ by
Fig. 1 Illustration of the transfer of an exemplary resulting flap force $F_{\text {flap }}$ and displacement $D_{\text {flap }}$ between the deflected CFD and undeflected CSM grid

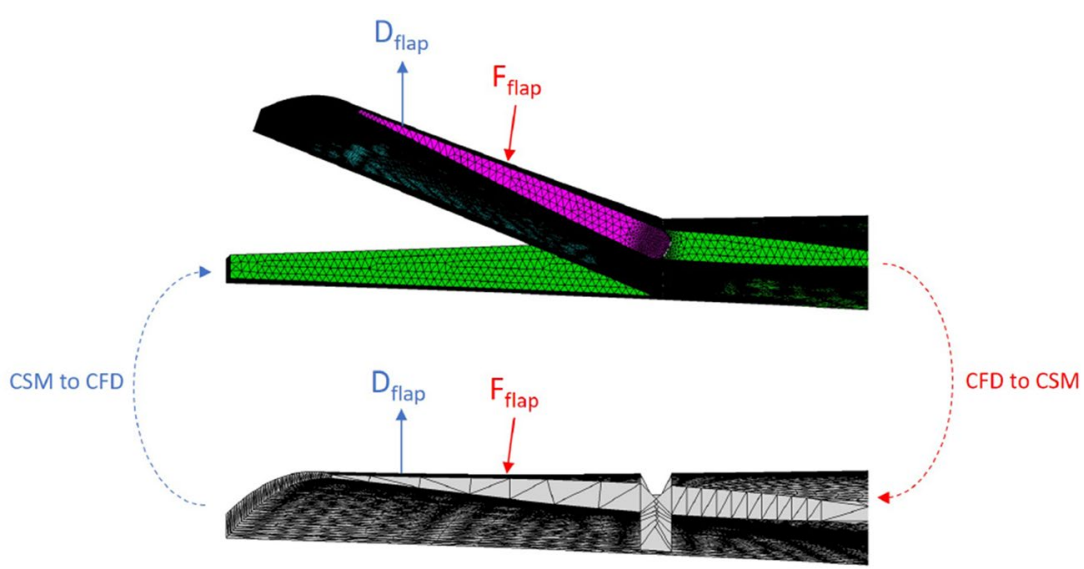




$$
\mathbf{d}_{\mathrm{s}}=\boldsymbol{\Phi}_{\mathrm{s}} \mathbf{q}
$$

The steady governing equation of a linear structure reads

$\mathbf{K} \mathbf{d}_{\mathrm{s}}=\mathbf{f}_{\mathrm{s}}$,

where $\mathbf{K}$ is the stiffness matrix, $\mathbf{d}_{\mathrm{s}}$ is the structural displacement vector and $\mathbf{f}_{\mathrm{s}}$ is the structural force vector. Expressed in generalized coordinates, Eq. (2) yields

$\boldsymbol{\Omega} \mathbf{q}=\boldsymbol{\Phi}_{\mathrm{s}}^{T} \mathbf{f}_{\mathrm{s}}$,

where $\boldsymbol{\Omega}=\boldsymbol{\Phi}_{\mathrm{s}}^{T} \mathbf{K} \boldsymbol{\Phi}_{\mathrm{s}}$ is the modal stiffness matrix. For a mass-normalized modal matrix, the modal mass is equal to one for each eigenmode. Each entry of the modal stiffness matrix is defined by $\boldsymbol{\Omega}_{i}=\left(2 \pi f_{i}^{2}\right)$, where $f_{i}$ is the structural eigenfrequency.

\subsection{CFD-CSM equilibrium}

The static aeroelastic equilibrium is found by an iterative process shown in Fig. 2, which is based on the numerical methods introduced above. The CFD-CSM loop is repeated until the convergence criterion or the specified number of CFD-CSM cycles is reached for the initial flap deflection angle. If a series of flap deflections are specified, the flap Chimera blocks are rotated accordingly and the CFD-CSM loop continues. The loop terminates, when the equilibrium of the final flap deflection angle is computed. This coupling process enables the efficient computation of the aeroelastic equilibrium for a series a flap deflection angles, as less iterations are needed for subsequent flap angles compared to the initial angle due to the better initial guess of the flow field and deformations. For the aeroelastic simulations in this work, four to five CFD-CSM exchange cycles are specified for each flap deflection angle.

\section{Model53 delta wing}

The numerical analysis in this work is performed for the Model53 test case, which is a cropped delta wing with a leading edge sweep angle of $\phi_{\mathrm{le}}=53^{\circ}$ and a trailing-edge sweep angle of $\phi_{\mathrm{te}}=3^{\circ}$. In its full-scale configuration, the root chord length measures $c_{\mathrm{r}}=8.4 \mathrm{~m}$ and the semi-span $s=5.1 \mathrm{~m}$. The wing is twisted up to $-4^{\circ}$ at the wing tip. The leading edge slat is constantly deployed to $20^{\circ}$ in this work, because such a configuration is common for delta wings at high angle of attack [23]. An inner and outer trailing edge flap are attached to the wing, which are being deflected from $\delta=-30^{\circ}$ to $\delta=30^{\circ}$ in the upcoming analysis. The leading edges of the inner and outer flap are both constantly positioned at $x / c_{\mathrm{r}}=0.88$ in chordwise direction over the span. Each flap has a span of $0.49 s=2.5 \mathrm{~m}$. The pitching moment reference point is located at $x / c_{\mathrm{r}}=0.5$. An overview of the geometric dimensions of the Model53 is shown in Fig. 3.

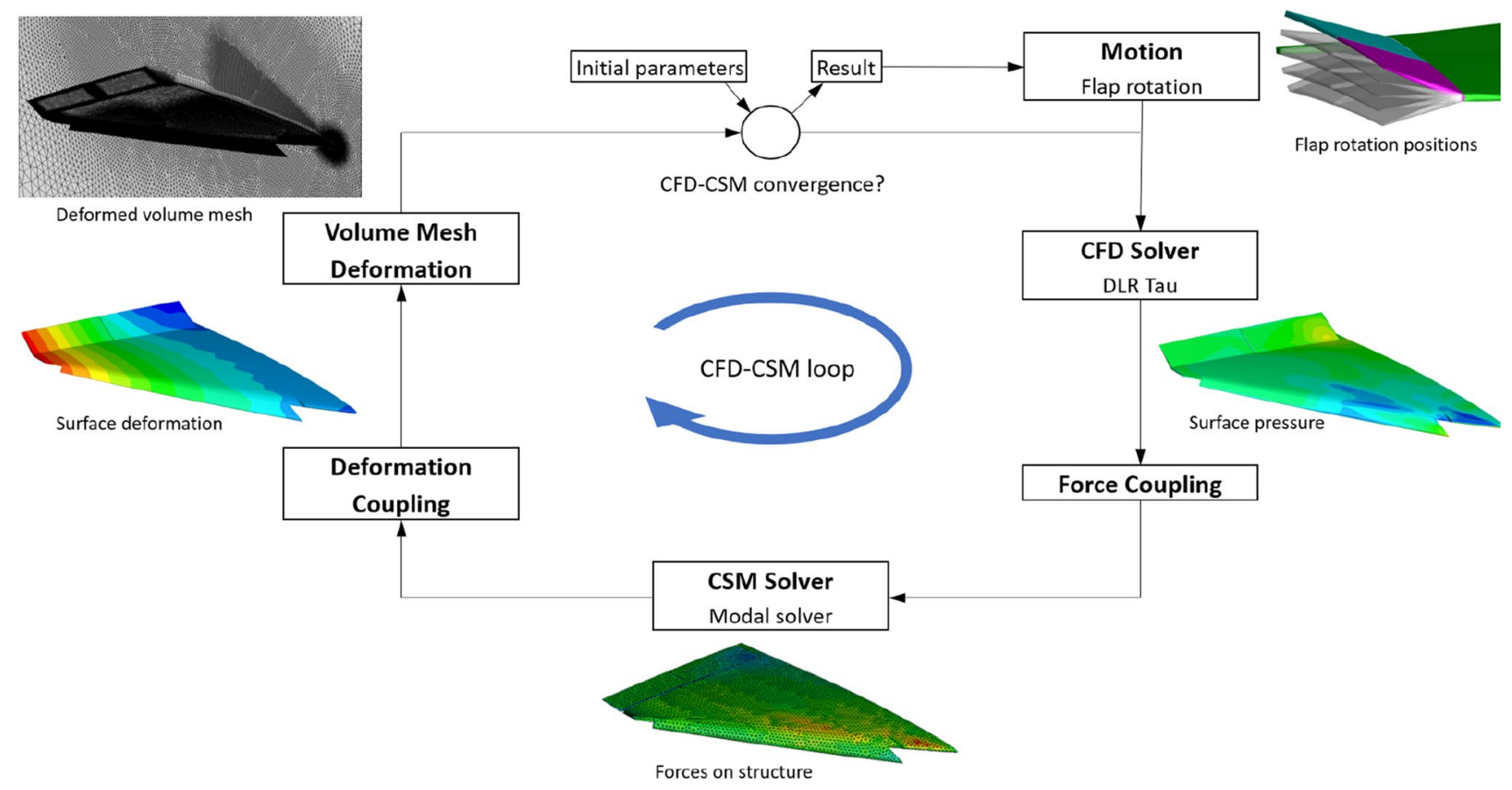

Fig. 2 CFD-CSM coupling process in the SimServer environment 


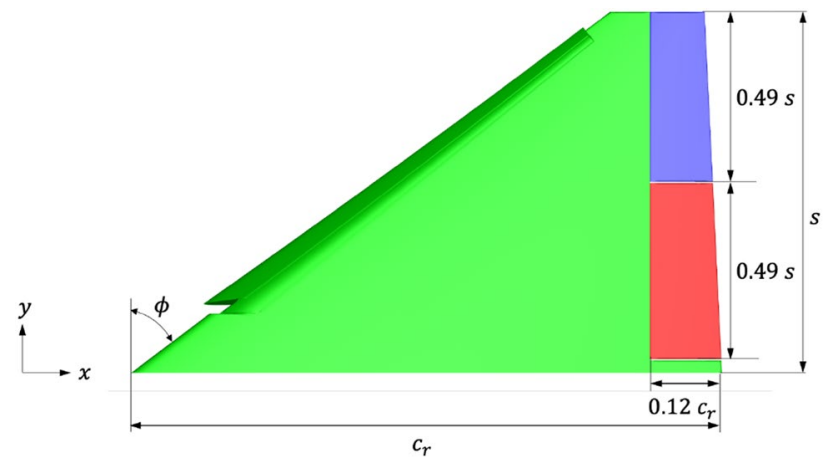

Fig. 3 Model53 dimensions, top view

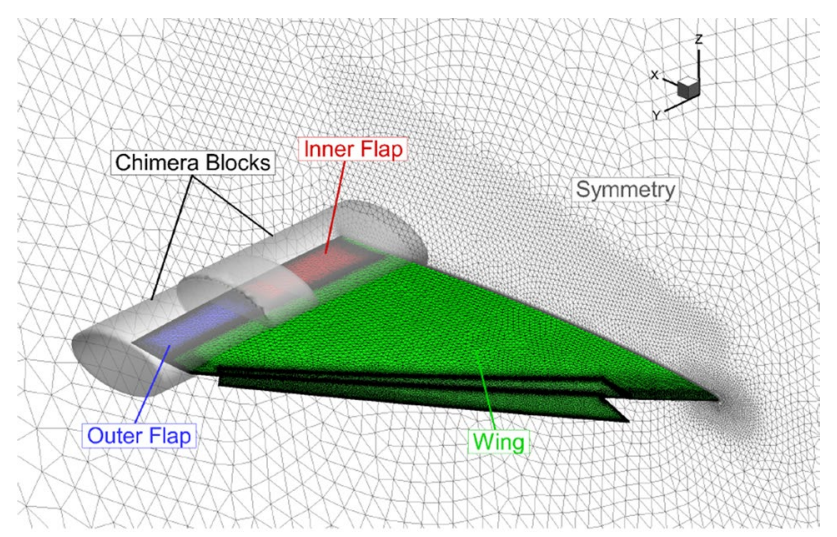

(a) Aerodynamic model and grid.

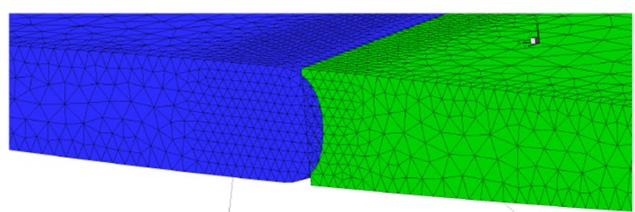

(b) Flap-wing intersection.

Fig. 4 Aerodynamic model and grid of the Model53 wing consisting of 7 million nodes

\subsection{Aerodynamic model}

Figure 4a shows the CFD model and CFD grid of the Model53. The inner and outer flap are treated as independent Chimera blocks which intersect with the main wing, Fig. 4b. A hybrid-unstructured grid generated with CENTAUR is used for the RANS computations, where prismatic cells are employed in boundary layer regions and unstructured cells in the remaining domain. A boundary layer mesh resolution with $y^{+} \leq 1$ is employed. A stretching factor of 1.27 is applied to a total of 35 prism layers. The computational grid is refined around the wing edges and in vortex dominated regions, see Fig. 4a. Compared to the wing edges, intersecting regions between the flaps and the main wing are further refined, to keep the Chimera interpolation error small, Fig. 4b. Following a grid convergence study, the chosen grid for the numerical simulations has approximately 7 million nodes. The difference in lift and pitching moment coefficient values was less than $2 \%$ in comparison to two finer grids with 15 and 32 million nodes. A sufficiently large hemispherical farfield boundary with a diameter of 100 reference chord lengths is chosen.

\subsection{Finite element model}

The FE model of the Model53, Fig. 5a, consists of 7928 grid points, 17,147 triangle elements and 24 bar elements. For the modeling of the ribs, spars, stiffening elements (shown in turquoise in Fig. 5a) and skin, shell elements are used. Stiffening elements are explicitly modeled and are employed to keep buckling fields sufficiently small as well as to reduce local eigenmodes. The inner and outer flap are structurally modeled with skin, ribs and spars and are attached to the wing with a hinge concept introduced in Ref. [24], which is shown in Fig. 5b for the Mode153. Each attachment consists of two triangle elements, four bar elements and a double node connecting the bar and triangle elements of the wing and flap side with a rigid body element. The inner and outer flap are each connected to the main wing with three attachments, of which one attachment can transfer translational and rotational degrees of freedom and two attachments only translational degrees of freedom. Each triangle element of the flap attachments is either connected to a spar of the main wing or the flap. The stiffness of the attachment can be controlled with the bar's material properties. This hinge concept

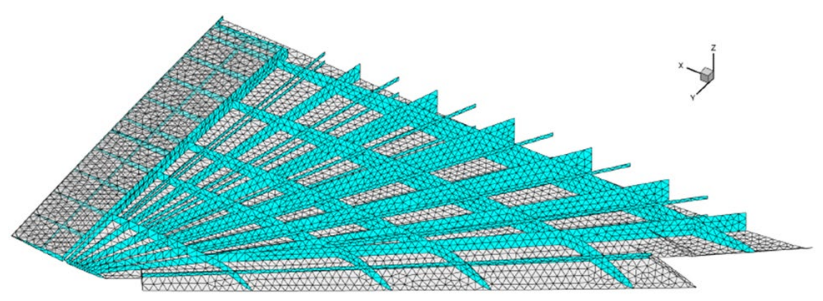

(a) Model53.

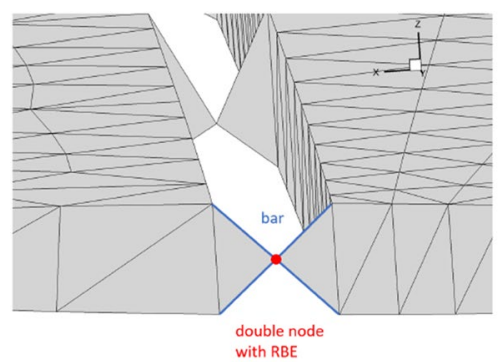

(b) Hinge concept.

Fig. 5 Structural model and hinge concept of the Model53 wing configuration 
Fig. 6 First four eigenmodes of the Model53 wing configuration

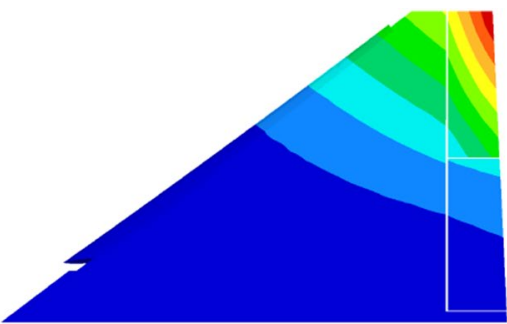

(a) 1 st mode $f_{1}=16.9 \mathrm{~Hz}$.

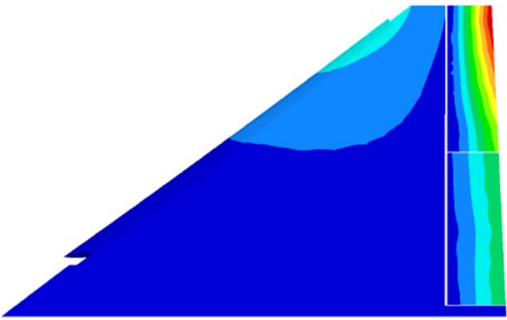

(c) 3rd mode $f_{3}=34.3 \mathrm{~Hz}$.

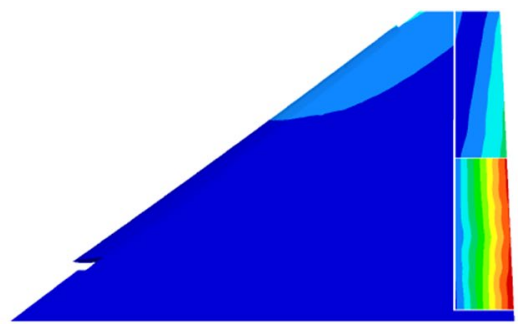

(b) 2nd mode $f_{2}=33.4 \mathrm{~Hz}$.

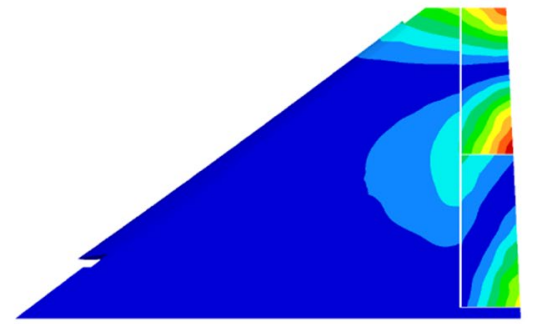

(d) 4 th mode $f_{4}=51.7 \mathrm{~Hz}$. enables a realistic behavior and physically meaningful analysis of control surface loads [8].

For the coupling, the 25 lowest mode shapes are used for the modal basis, since higher modes are dominated by buckling effects. The first four modes are shown in Fig. 6. The first bending mode occurs at $f_{1}=16.9 \mathrm{~Hz}$ and the first torsional mode is split in two frequencies $f_{2}=33.4 \mathrm{~Hz}$ and $f_{3}=34.3 \mathrm{~Hz}$, where the lower frequency contains a larger contribution of the inner flap movement and the higher frequency a larger contribution of the outer flap movement around their hinge axis. The second bending is observable at the fourth natural frequency $f_{4}=51.7 \mathrm{~Hz}$. Clearly, the individual movements of the inner and outer flap with respect to the main wing are contained in the set of 25 modes. This is important for a meaningful analysis of the influence of flexibility effects from the control surfaces. The wing is clamped at the root and inertial forces are not considered. For all structural components, aluminum has been chosen as material. A mesh independence study for the structural grid has been performed and revealed deviations smaller than $1 \%$ compared to the finer grid $(18,499$ nodes, 38,823 triangle elements) in terms of the natural frequencies and corresponding eigenvectors.

\section{Computational results}

\subsection{Flow field description}

Before investigating the influence of flexibility on the aerodynamic characteristics of the Model53, the flow field is computed assuming the wing to be fully rigid. To verify the robustness of the presented flexible Chimera method, high dynamic pressures and angles of attack are targeted where larger deformations are expected. Thus, the computations are performed for freestream values of $M a=0.8, \alpha=\left[10^{\circ}, 15^{\circ}, 20^{\circ}, 25^{\circ}\right]$ at sea level, which leads to a dynamic pressure and Reynolds number of $q=45 \mathrm{kPa}$, $R e=15.1 \times 10^{7}$ with the root chord as reference length.

Figure 7 depicts the flow field around the Model53 wing exemplarily for two angles of attack $\alpha=\left[10^{\circ}, 20^{\circ}\right]$ and no flap deflection $\delta=0^{\circ}$. For an angle of attack $\alpha=10^{\circ}$, the $x$-vorticity slice contours and the surface pressure distribution indicate the formation of a weak leading-edge vortex, which separates near the apex. Supersonic flow can be observed in the vicinity of the apex. Additional to the apex vortex, a wing tip vortex forms at the leading-edge outboard of the slat. At the transition from the slat to the main wing, a thin low-pressure region, extending from approximately $40-80 \%$ of the wing-span is present in the surface pressure distribution. Here, supersonic speeds are reached locally. This region of supersonic flow is terminated by a shock wave, which causes the boundary layer to separate from the wing's surface downstream of the shock. The separated flow downstream of the shock is indicated by higher vorticity values, Fig. 7a. At this angle of attack, supersonic flow is limited to the aforementioned regions near the slat-wing transition and the apex.

At $\alpha=20^{\circ}$, the complexity of the flow field around the Model53 wing increases. Compared to $\alpha=10^{\circ}$, a stronger apex vortex located further inboard is observable from the more pronounced suction footprint in the pressure distribution as well as higher vorticity values in the slice contours, Fig. 7b. In addition, large regions of supersonic flow are present above the upper wing surface in comparison to $\alpha=10^{\circ}$, Fig. 7c. The highest Mach number is reached in 
Fig. 7 Surface pressure distribution $C_{p}$ including $x$-vorticity magnitude $\omega_{\mathrm{x}}$ and Mach number contours Ma for the Model53 at Ma $=0.8$, $\operatorname{Re}=15.1 \times 10^{7}, \alpha=10^{\circ}, 20^{\circ}$ and $\delta=0^{\circ}$

the apex vortex core with $M a_{\max } \approx 2$. Further downstream, a weaker suction footprint and lower vorticity values are present, which can be related to the bursting of the apex vortex at $x / c_{\mathrm{r}} \approx 0.2$. The breakdown of the apex vortex is influenced by complex shock/vortex interactions and occurs downstream of an initial shock, visible in the pressure distribution, Fig. 7c. Downstream of the apex vortex breakdown, the vortical flow is subsonic and additional shocks occur inboard of the vortex, which are clearly visible through the pressure distribution on the symmetry plane, Fig. 7c.

A second vortex separates at the deployed slat. Due to the smaller local angle of attack resulting from the slat deployment, the slat vortex has a different, rather wake-type, topology compared to the apex vortex. The breakdown position of the slat vortex is located in the rear half of the wing and is indicated by low vorticity magnitudes as well as reverse axial flow $u / U_{\infty}<0$, Fig. 7b. Similar to the apex vortex, the formation and breakdown of the slat vortex is significantly influenced by shock/vortex interactions. Additionally, an interaction between both vortices is observable from the vorticity contours in Fig. $7 \mathrm{~b}$.

\subsection{Influence of flexibility}

General trends Figure 8 shows the lift coefficient $C_{\mathrm{L}}$ and the lift coefficient deviation $\Delta C_{\mathrm{L}}=C_{\mathrm{L} \text {,flexible }}-C_{\mathrm{L} \text {,rigid }}$ over several flap deflection angles. Accordingly, in Fig. 9 the pitching moment coefficient $C_{\mathrm{My}}$ and deviation $\Delta C_{\mathrm{My}}=C_{\mathrm{My} \text {,flexible }}-C_{\mathrm{My}, \text { rigid }}$ is depicted. The results of the rigid configuration have been obtained by CFD simulations, whereas the results for the flexible wing have been performed with the aeroelastic Chimera method described in Sect. 2. For the rigid and flexible results, the general trend is that the lift coefficient $C_{L}$ increases with increasing flap deflection angles for all angles of attack, except at $\alpha=15^{\circ}$ from $\delta=20^{\circ}$ to $\delta=30^{\circ}$ and at $\alpha=25^{\circ}$ from $\delta=-30^{\circ}$ to $\delta=-20^{\circ}$, Fig. 8a. The gain in lift coefficient gets smaller for each $\Delta \alpha=5^{\circ}$ increase in angle of attack, which means that $C_{\mathrm{L}}$ has a sub-linear behaviour with respect to $\alpha$ for the given configuration and flow conditions. The pitching moment coefficient $C_{\mathrm{My}}$ decreases from $\delta=-30^{\circ}$ to $\delta=30^{\circ}$ for all angles of attack, except for $\alpha=25^{\circ}$ from $\delta=-30^{\circ}$ to $\delta=-20^{\circ}$, Fig. 9a, b. Starting from the baseline of $\delta=0^{\circ}$, the highest lift and pitching moment coefficient increments, or highest aerodynamic control surface efficiency, can be achieved within $-10^{\circ}<\delta<10^{\circ}$ for all angles of attack. Both curves flatten towards the minimum and maximum deflection angle.

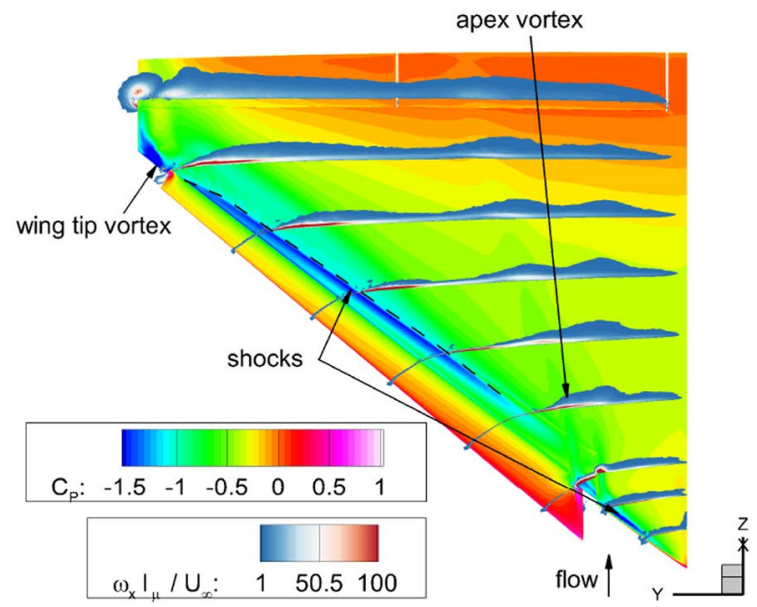

(a) $\alpha=10^{\circ}, \delta=0^{\circ}$.

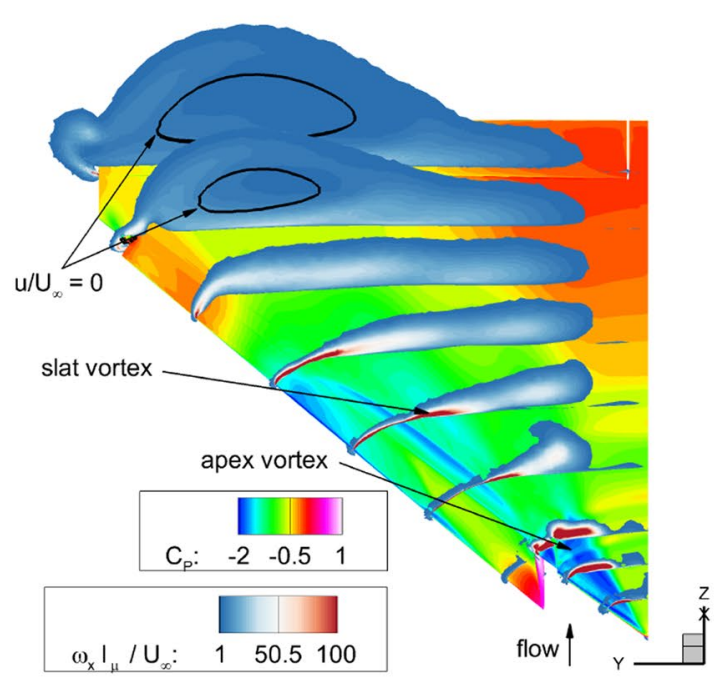

(b) $\alpha=20^{\circ}, \delta=0^{\circ}$.

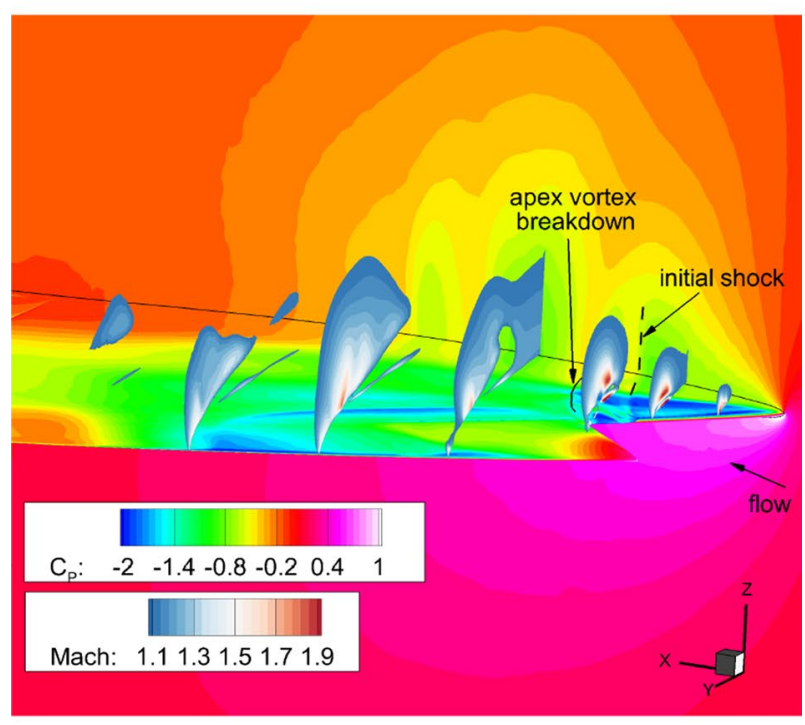

(c) $\alpha=20^{\circ}, \delta=0^{\circ}$. 


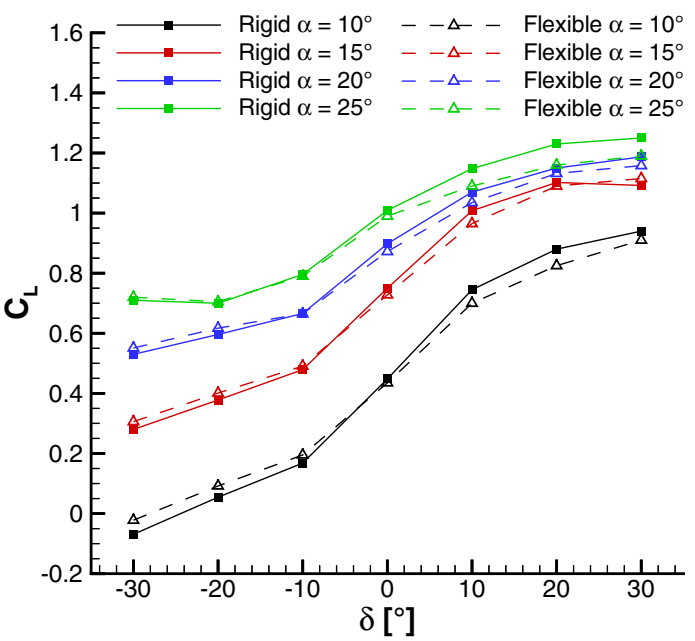

(a) Lift coefficient $C_{L}$ for $\alpha=\left[10^{\circ}, 15^{\circ}, 20^{\circ}, 25^{\circ}\right]$.

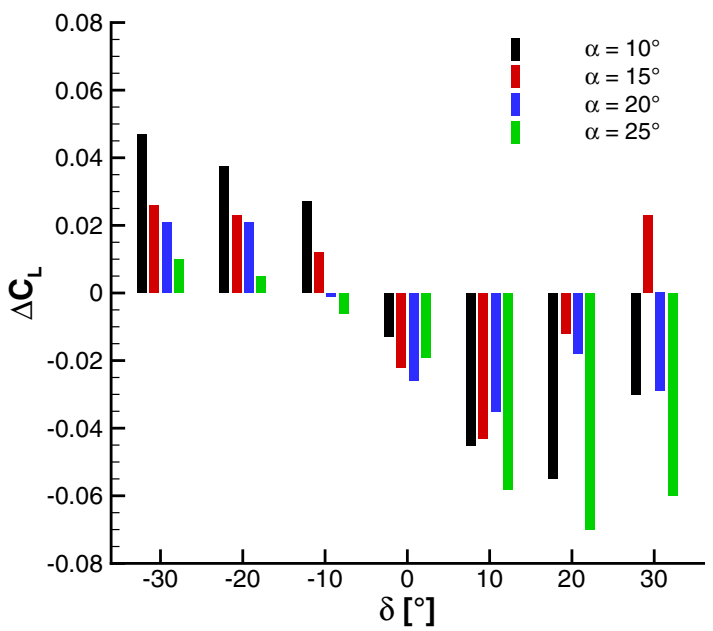

(b) Lift coefficient deviation $\Delta C_{L}=C_{L}$, flexible $-C_{L, \text { rigid }}$ for $\alpha=\left[10^{\circ}, 15^{\circ}, 20^{\circ}, 25^{\circ}\right]$.

Fig. 8 Lift coefficient $C_{\mathrm{L}}$ and deviation $\Delta C_{\mathrm{L}}$ over flap deflection angle $\delta$ for $\mathrm{Ma}=0.8, \mathrm{Re}=15.1 \times 10^{7}$ and $\alpha=\left[10^{\circ}, 15^{\circ}, 20^{\circ}, 25^{\circ}\right]$

The wing's flexibility has a varying influence on the lift and pitching moment coefficient characteristics over the flap deflection angles dependent on the angle of attack. In general, the trend is that the lift coefficient decreases for $\delta \geq 0^{\circ}$ and increases for $\delta<0^{\circ}$ compared to the rigid configuration, whereas the pitching moment increases for $\delta \geq 0^{\circ}$ and decreases for $\delta<0^{\circ}$, Figs. 8 and 9. Furthermore, the offset in lift and pitching moment coefficient decreases with higher angle of attack for upward deflections $\delta<0^{\circ}$. For downward deflections and no deflection $\delta \geq 0^{\circ}$, the offsets increase for $\alpha=25^{\circ}$ compared to $\alpha=10^{\circ}$, however, in between no clear trend can be identified. To understand, which changes in the flow field lead to the differences in the lift and pitching moment coefficient characteristics between the rigid and flexible case, the surface pressure distribution is analyzed

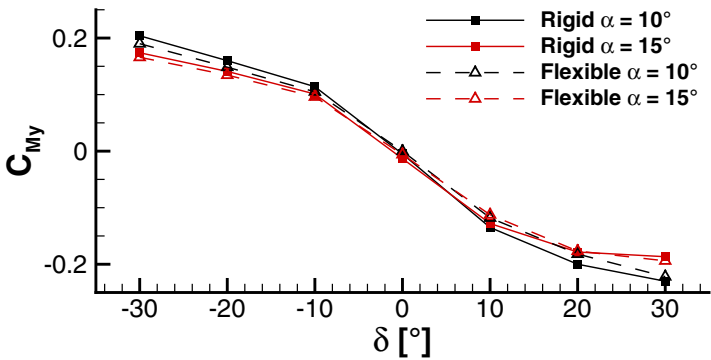

(a) Pitching moment coefficient $C_{M y}$ for $\alpha=\left[10^{\circ}, 15^{\circ}\right]$.

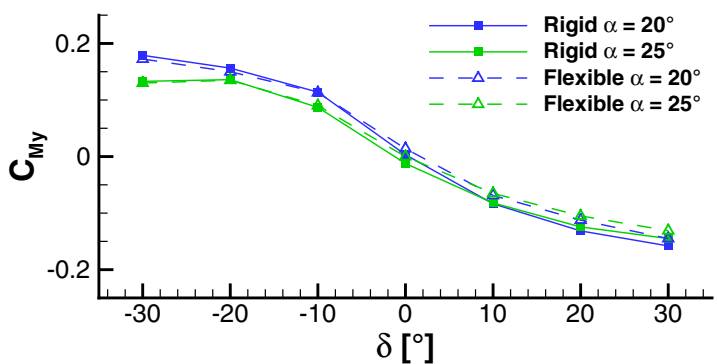

(b) Pitching moment coefficient $C_{M y}$ for $\alpha=\left[20^{\circ}, 25^{\circ}\right]$.

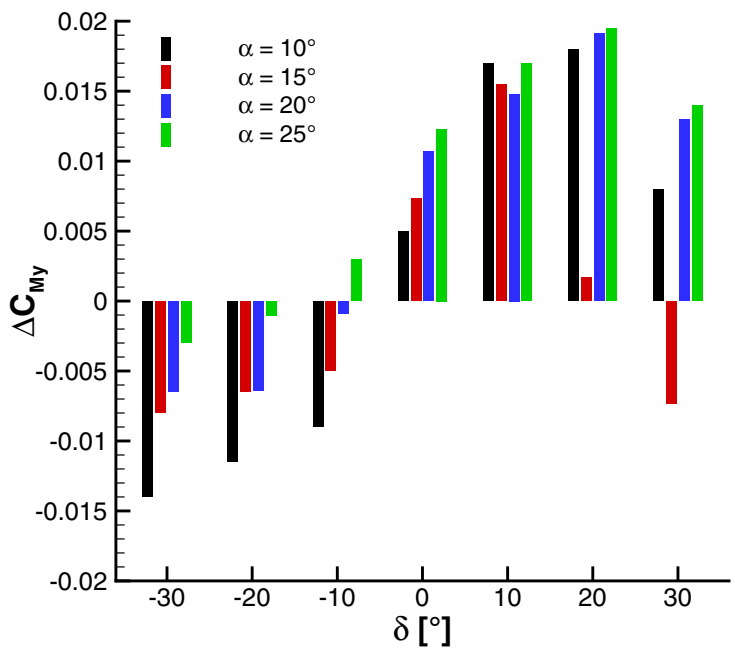

(c) Pitching moment coefficient deviation $\Delta C_{M y}=$ $C_{M y, \text { flexible }}-C_{M y, \text { rigid }}$ for $\alpha=\left[10^{\circ}, 15^{\circ}, 20^{\circ}, 25^{\circ}\right]$.

Fig. 9 Pitching moment coefficient $C_{\mathrm{My}}$ and deviation $\Delta C_{\mathrm{My}}$ over flap deflection angle $\delta$ for $\mathrm{Ma}=0.8, \mathrm{Re}=15.1 \times 10^{7}$ and $\alpha=\left[10^{\circ}, 15^{\circ}, 20^{\circ}, 25^{\circ}\right]$

for both. Figure 10 depicts the rigid wing on the left and the flexible wing in a mirrored view on the right for selected angles of attack and flap deflection angles. The $z$-displacement component of the same selection of angles of attack and flap deflection angles is shown in Fig. 11.

Angle of attack $\alpha=10^{\circ}$ : At $\alpha=10^{\circ}$ and a flap deflection of $\delta=0^{\circ}$, the flexible wing provides a lower lift coefficient value compared to the rigid wing, Fig. 8. This results from the reduced local angle of attack due to increased wing twist 
of the flexible wing. The absolute offset in lift coefficient and pitching moment coefficient increases for upward deflected flaps $\delta<0^{\circ}$ as well as for downward deflected flaps $\delta>0^{\circ}$ compared to the non-deflected configuration $\delta=0^{\circ}$, see Fig. 8.

For upward flap deflections, the main driver for the offset in force and moment coefficient of the flexible wing can be attributed to a differing effective flap deflection angle. Due to the wing's flexibility, the effective flap deflection angle at the wing tip is $\delta_{\text {eff }}=-27.8^{\circ}$ for a rigid upward deflection of $\delta=-30^{\circ}$. The reduced effective flap deflection, results in lower pressure levels on the suction side, which indicate higher lift, of the flexible wing compared to the rigid one, Fig. 10a. From the displacement contours, it is apparent that the wing is deformed downwards, Fig. 11a, as the overall configuration generates negative lift due to the high downforce from the flaps, Fig. 8a. The maximum displacement for $\delta=-30^{\circ}$ is $d_{z} \approx-0.05 \mathrm{~m}$ at the outer flap's wing tip.

For a downward deflection of $\delta=30^{\circ}$, the most apparent difference in pressure distribution is the increased suction footprint of the wing-tip vortex present on the flexible configuration, which is a result of the deformed wing geometry that enables the flow to maintain supersonic speeds further aft, Fig. 10b. The effective flexible flap deflection angle is $\delta_{\text {eff }}=25.8^{\circ}$ for a rigid downward deflection of $\delta=30^{\circ}$. In contrast to the upward flap deflection $\delta=-30^{\circ}$, a larger contribution of wing twist can be seen for $\delta=30^{\circ}$, Fig. 11b. A reduction in local angle of attack due to wing twist as well as lower effective flap deflection angles contribute to lower lift and higher pitching moment coefficient values for downward deflected flaps. Even though the largest displacements and biggest difference in effective deflection angle $\Delta \delta$ occurs at $\delta=30^{\circ}$, the highest offset $\Delta C_{\mathrm{L}}$ and $\Delta C_{\mathrm{My}}$ is reached at $\delta=20^{\circ}$, because the flow around the main wing is more sensitive to effective flap deflection angle changes at $\delta=20^{\circ}$. A similar course for the absolute pitching moment deviations over the flap deflection angles can be seen in Fig. 9c.

Angle of attack $\alpha=15^{\circ}$ With a higher angle of attack $\alpha=15^{\circ}$, the offset in lift and pitching moment coefficient between the rigid and flexible configuration rises at the zero flap deflection angle $\delta=0^{\circ}$ compared to $\alpha=10^{\circ}$, Figs. 8 and 9. This is caused by a higher wind loading which leads to larger displacements and hence to a greater reduction in lift and increase in pitching moment coefficient.

Compared to the other angles of attack, an interesting tendency can be seen at $\alpha=15^{\circ}$ for downward deflections, where the deviation in lift and pitching moment coefficient changes its sign at $\alpha=15^{\circ}$ from negative $\Delta C_{\mathrm{L}}$ and positive $\Delta C_{\mathrm{My}}$ at $\delta=20^{\circ}$, to positive $\Delta C_{\mathrm{L}}$ and negative $\Delta C_{\mathrm{My}}$ at $\delta=30^{\circ}$, Figs. $8 \mathrm{~b}$ and $9 \mathrm{c}$. From the surface pressure distribution in Fig. 10c, d, it is evident that the decrease of lift for the rigid wing is caused by an upstream movement of the breakdown position of the slat vortex from $\delta=20^{\circ}$ to $\delta=30^{\circ}$. Concerning the influence of flexibility, a further downstream breakdown position of the slat vortex can be seen for $\delta=20^{\circ}$ and $\delta=30^{\circ}$, even though the displacement contours for both flap deflection angles do not differ significantly, Fig. 11c, d.

Angle of attack $\alpha=\mathbf{2 0}^{\circ}$ In accordance with the decreasing offset in lift coefficient between the rigid and flexible configuration from $\alpha=10^{\circ}$ to $\alpha=20^{\circ}$ for upward flap deflection angles $\delta<0^{\circ}$, Figs. 8 and 9, the pressure distribution contours differ only marginally for $\alpha=20^{\circ}$, Fig. 10e, f. The reason is that for $\delta<0^{\circ}$ and increasing angles of attack, the downward force or efficiency of the flaps decreases, since the flap's upper sides encounter wake type flow conditions with decreased pressure levels. Less down-force acting on the flaps leads to a smaller difference between the effective flexible and rigid deflection angle and hence a smaller offset in lift and pitching moment coefficient. The effective angle of attack is $\delta_{\text {eff }}=-29^{\circ}$ for a rigid upward deflection of $\delta=-30^{\circ}$. From the displacement contours, Fig. 11e, it is apparent that the contour lines are more aligned with the $\mathrm{x}$-axis, which relates to a small amount of twist. Concerning downward flap deflections in case of $\alpha=20^{\circ}$, the difference between effective and rigid flap deflection angle is $\delta_{\text {eff }}=26.7^{\circ}$ for $\delta=30^{\circ}$. Additionally, wing twist is more present for $\delta \geq 0^{\circ}$ which combined with smaller effective flap deflection angles explains the reduction in lift and increase in pitching moment coefficient values shown in Figs. 8a and 9a. The maximum deformation for $\delta=30^{\circ}$ decreased from $d_{z, \text { max }} \approx 0.2 \mathrm{~m}$ at $\alpha=10^{\circ}$ to $d_{z, \text { max }} \approx 0.18 \mathrm{~m}$ at $\alpha=20^{\circ}$ due to the mentioned lower flap loading.

Angle of attack $\alpha=25^{\circ}$ For $\alpha=25^{\circ}$ and $\delta=0^{\circ}$, a modest upstream movement of the apex vortex breakdown position in case of the flexible configuration is evident from the pressure distribution contours in Fig. 10g. In contrast, a significant difference with regard to the apex vortex bursting location is apparent for $\delta=30^{\circ}$ in Fig. 10h. On the one hand, the breakdown position moves further downstream and increases lift near the apex of the flexible wing. On the other hand, higher pressure levels can be seen inboard of the wing resulting from a stronger shock wave as well as higher pressure levels outboard of the wing due to wing twist. In combination with reduced effective flap deflections, lift is reduced significantly for $\alpha=25^{\circ}$ and $\delta>0^{\circ}$ compared to the rigid configuration. The effective flap deflection angle is $\delta_{\text {eff }}=26.4^{\circ}$ for a rigid downward deflection of $\delta=30^{\circ}$. Even though the overall deformations are similar to $\alpha=20^{\circ}$, compare Fig. 11f, g, the influence of flexibility on the force and pitching moment coefficient is considerably different.

Summary The differences in lift and pitching moment coefficient over the range of flap deflection angles, shown in Figs. 8 and 9, can be attributed to a change of the effective flap deflection angle for upward flap deflections $\delta<0^{\circ}$ as well as to an additional increasing contribution of wing 


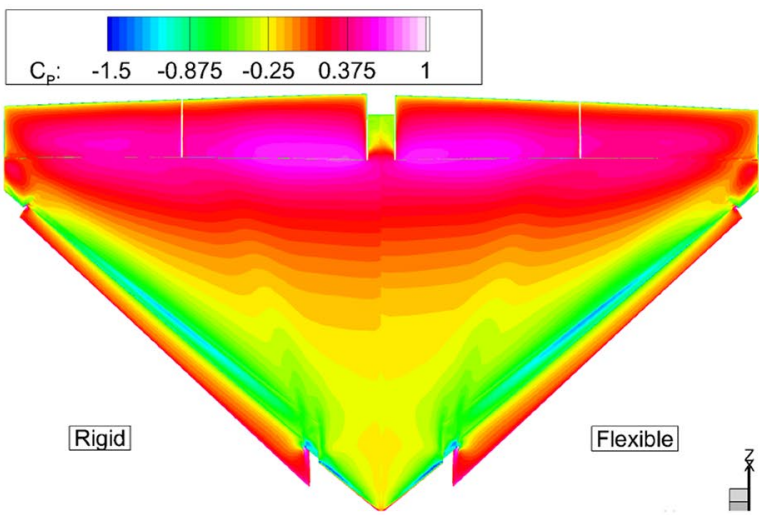

(a) $\alpha=10^{\circ}$ and $\delta=-30^{\circ}$.

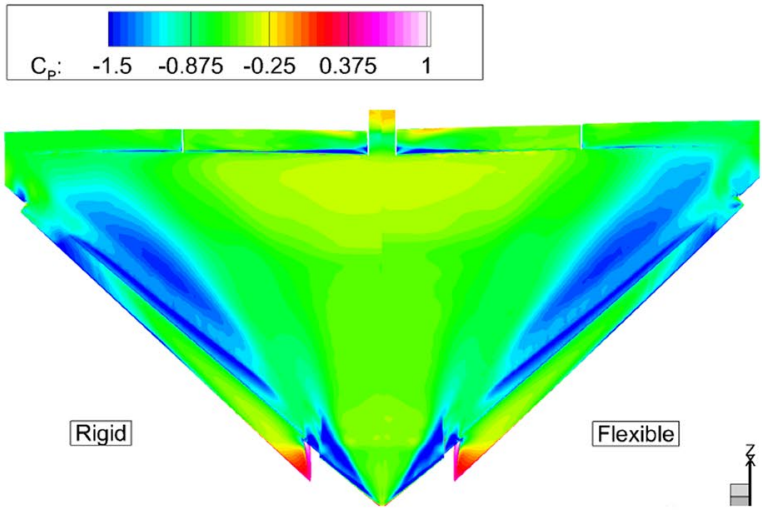

(c) $\alpha=15^{\circ}$ and $\delta=20^{\circ}$.

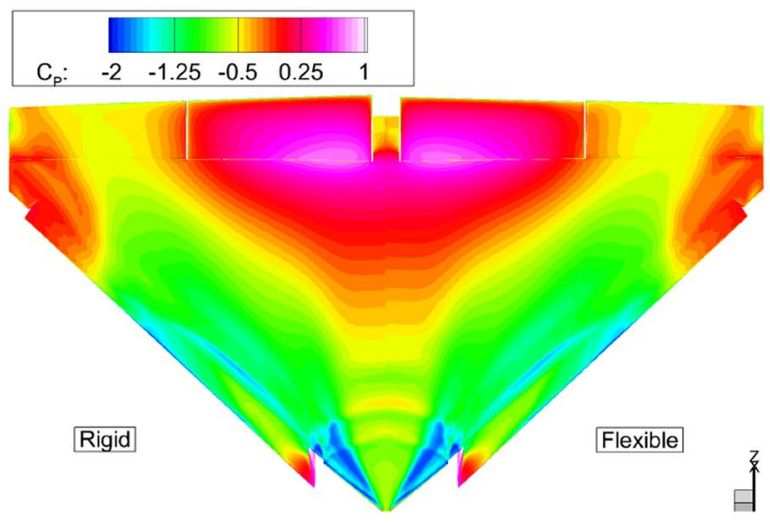

(e) $\alpha=20^{\circ}$ and $\delta=-30^{\circ}$.
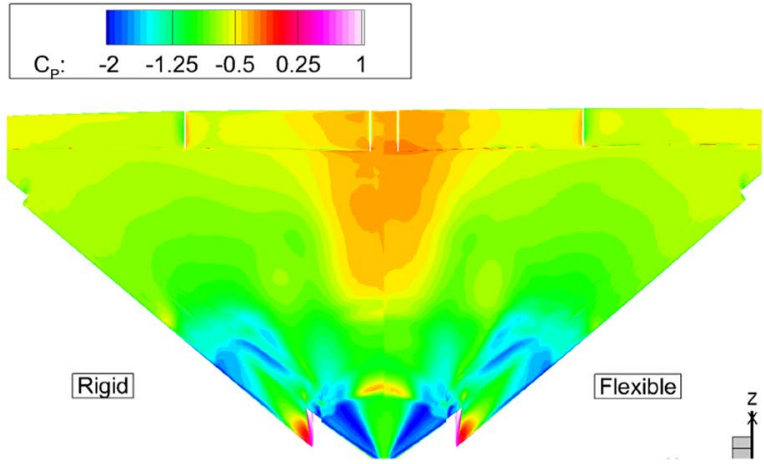

(g) $\alpha=25^{\circ}$ and $\delta=0^{\circ}$.
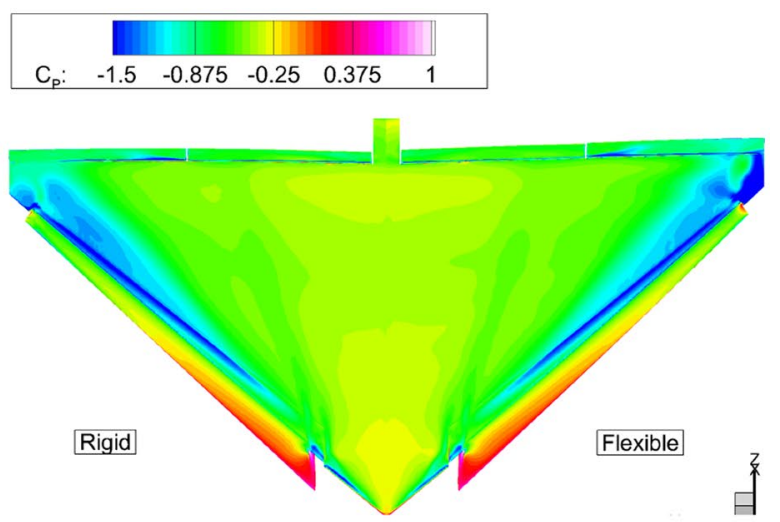

(b) $\alpha=10^{\circ}$ and $\delta=30^{\circ}$.
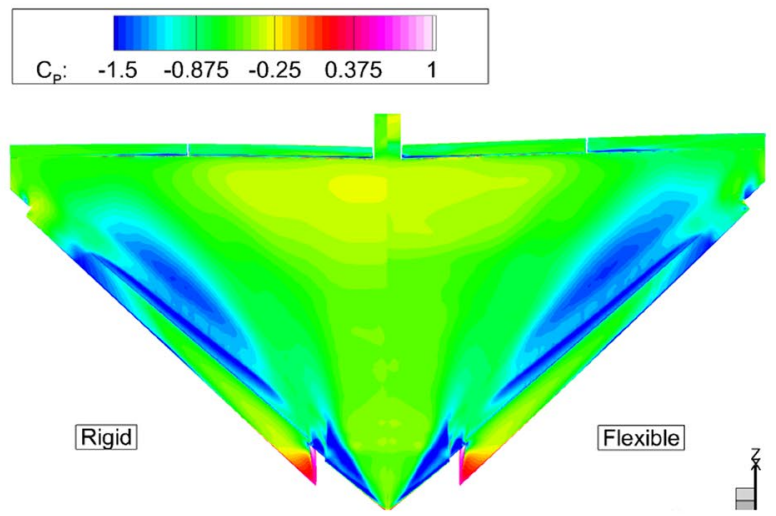

(d) $\alpha=15^{\circ}$ and $\delta=30^{\circ}$.
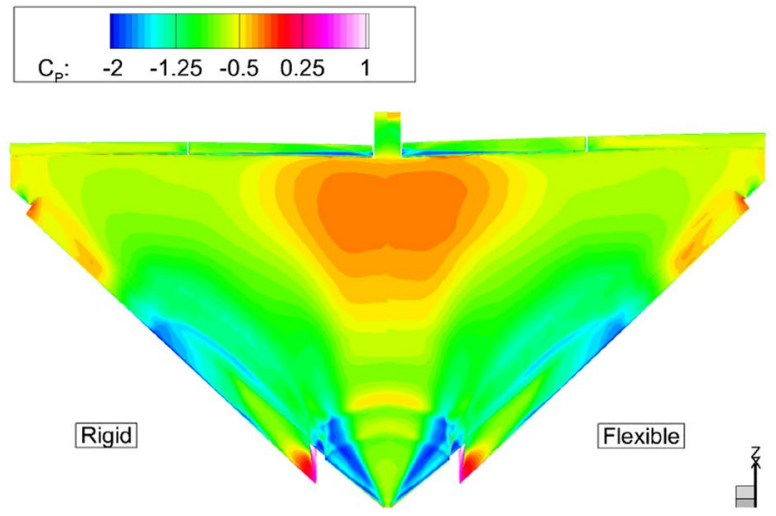

(f) $\alpha=20^{\circ}$ and $\delta=30^{\circ}$.
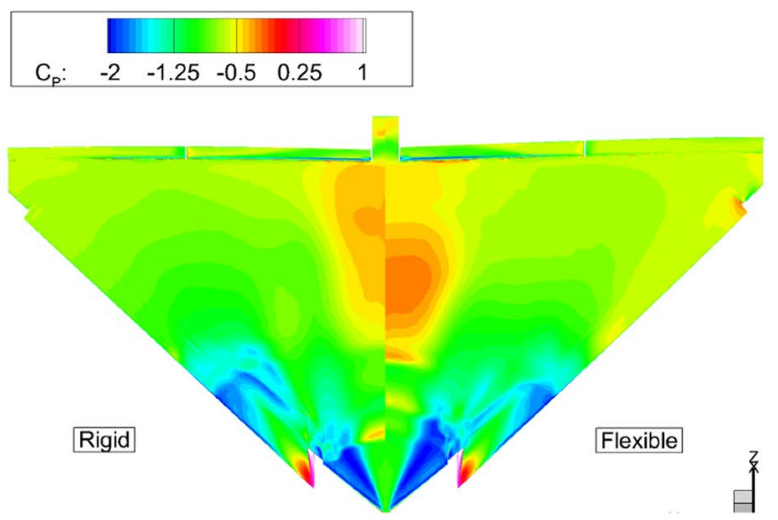

(h) $\alpha=25^{\circ}$ and $\delta=30^{\circ}$. 
4Fig. 10 Surface pressure coefficient distribution $C_{\mathrm{p}}$ on the upper side of the Model53 for the rigid and flexible configuration at $\mathrm{Ma}=0.8$, $\operatorname{Re}=15.1 \times 10^{7}, \alpha=\left[10^{\circ}, 15^{\circ}, 20^{\circ}, 25^{\circ}\right]$ and selected flap deflection angles $\delta$

twist for flap deflection angles $\delta \geq 0^{\circ}$. The increase in lift and reduction in pitching moment coefficient values for $\delta<0^{\circ}$ decreases with higher angles of attack, as the flaps generate less down-force which leads to a smaller deviation of the rigid and flexible flap deflection angle. The maximum offset for upward deflections occurs at $\alpha=10^{\circ}$ and $\delta=30^{\circ}$ with $\Delta C_{\mathrm{L}}=+0.047$ and $\Delta C_{\mathrm{My}}=-0.014$ compared to the rigid configuration. Starting from $\alpha=10^{\circ}$, the reduction in lift coefficient for flap deflections $\delta>0^{\circ}$ initially decreases until $\alpha=20^{\circ}$, as the overall deformation gets smaller which results in less lift generated by the flaps. However, at $\alpha=25^{\circ}$, the largest offset in lift and pitching moment coefficient is visible, even though the deformations are similar to $\alpha=20^{\circ}$. For downward deflections, the maximum offset relative to absolute values of the rigid wing occurs at $\alpha=25^{\circ}$ and $\delta=20^{\circ}$ with $\Delta C_{\mathrm{L}}=-0.07$ and $\Delta C_{\mathrm{My}}=+0.02$. Concerning the pitching moment coefficient offsets for downward flap deflections, it is difficult to provide a short summary of the trend, as the offsets are distributed non-uniformly over the angles of attack. Consequently, the non-linear influence of flexibility on the vortical flow field of the Model53 configuration highlights the necessity of high-fidelity CFD-CSM simulations to capture relevant flow phenomena correctly.

\subsection{Computational effort}

The completion of the aerodynamic simulation took 1500 CPU hours on Intel Xeon processors with a core frequency of $3.1 \mathrm{GHz}$. For the aeroelastic simulation, 2250 CPU hours have been used, which is 1.5 times more compared to the rigid case. Concerning the aeroelastic case, $92 \%$ of the computational time has been used for CFD solver iterations, 3\% for the volume grid deformation, $3 \%$ for the fluid-structure coupling and the remaining $2 \%$ for pre-processing and the creation of log files. The increase in computational time for the aeroelastic simulation is considered acceptable given the improvement in the accuracy of the results.

Figure 12 depicts the convergence history of the lift and pitching moment coefficient for the aerodynamic and aeroelastic simulation. In addition, the flap deflection angles for the corresponding iteration range is shown.

The iteration at which the rotation of the flap deflection angle is performed, is also clearly visible by identifying strong changes in the course of the lift or pitching moment coefficient. Compared to the initial flap deflection angle, a decrease in the amount of iterations can be seen for subsequent deflection angles. This highlights the advantage of using the Chimera technique to reduce computational time for the simulation of several flap deflections.

\section{Conclusion}

In this work, the developed numerical method for performing aeroelastic simulations including deformable control surfaces modeled through the Chimera technique was introduced and described. This method is based on the SimServer multi-disciplinary simulation environment. A fluid-structure coupling was developed which is suited to work in combination with the Chimera technique and the deflection of control surfaces. Furthermore, the aeroelastic simulation process has been adapted to include the rotation of flaps.

Aerodynamic and aeroelastic computations involving the Model53 delta wing configuration were performed at transonic speed and high dynamic pressure. The wing features a deployed slat and an inboard and outboard trailing edge flap. The flaps were deflected from $\delta=-30^{\circ}$ to $\delta=30^{\circ}$ using a Chimera mesh in the aerodynamic model. In the structural model, the flaps are attached to the main wing with a hinge concept that ensures a realistic modeling of the control surfaces. The complex flow field around the Model53 configuration at $M=0.8$ and $\operatorname{Re}=15.1 \times 10^{7}$ is characterized by the interaction of vortices and shock waves.

Aerodynamic computations under-predicted lift and over-predicted pitching moment values for upward flap deflections compared to aeroelastic computations. For each angle of attack, the offset between the rigid and flexible wing increased with higher upward flap deflections. With increasing angle of attack, the differences between the aerodynamic and aeroelastic results diminished as a result of decreasing flap down-force. The highest difference for upward flap deflections was observed at $\alpha=10^{\circ}, \delta=-30^{\circ}$ with $\Delta C_{\mathrm{L}}=+0.047$ and $\Delta C_{\mathrm{My}}=-0.014$ compared to the rigid wing $\left(C_{\mathrm{L} \text {,rigid }}=-0.07\right.$ and $\left.C_{\mathrm{My} \text {,rigid }}=0.204\right)$. The differences in lift and pitching moment are mainly driven by a change in the effective flap deflection angle, which for upward deflected flaps reaches the highest difference of $\Delta \delta=+2.2^{\circ}$ at $\alpha=10^{\circ}, \delta=-30^{\circ}$.

For downward flap deflections, lift was generally overpredicted and pitching moment under-predicted by the aerodynamic simulations. The deviation in lift and pitching moment coefficient generally increased with higher downward deflection angles, but decreased again after a certain deflection angle. Dependent on the angle of attack, the maximum offset in lift and pitching moment coefficient occurred at differing flap deflection angles, but within the range of $10^{\circ} \leq \delta \leq 20^{\circ}$. Starting from $\alpha=10^{\circ}$ the offset in lift coefficient values between the rigid and flexible configuration initially decreased until $\alpha=20^{\circ}$, but increased considerably at $\alpha=25^{\circ}$ even though the overall deformations and effective 


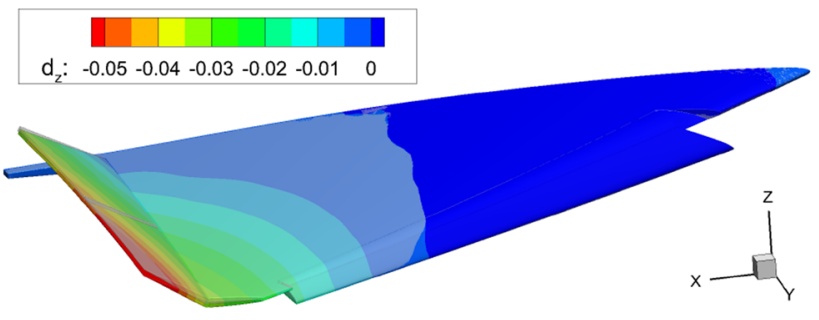

(a) $\alpha=10^{\circ}$ and $\delta=-30^{\circ}$.

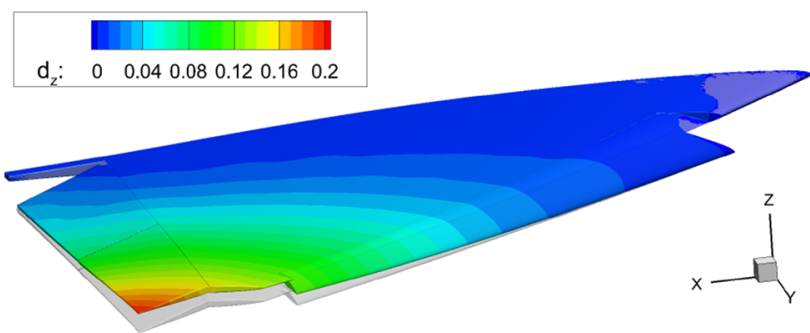

(c) $\alpha=15^{\circ}$ and $\delta=20^{\circ}$.

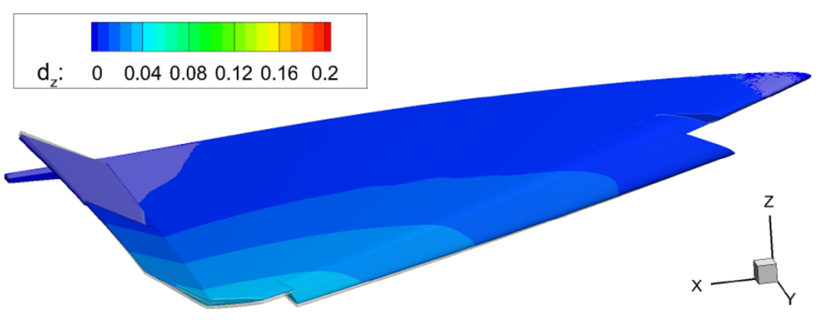

(e) $\alpha=20^{\circ}$ and $\delta=-30^{\circ}$.

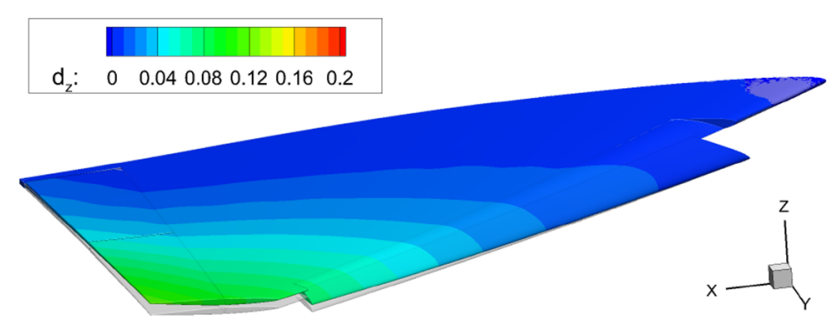

(g) $\alpha=25^{\circ}$ and $\delta=0^{\circ}$.

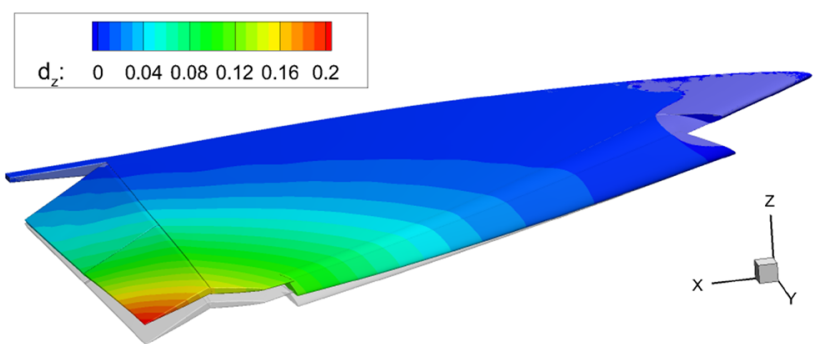

(b) $\alpha=10^{\circ}$ and $\delta=30^{\circ}$.

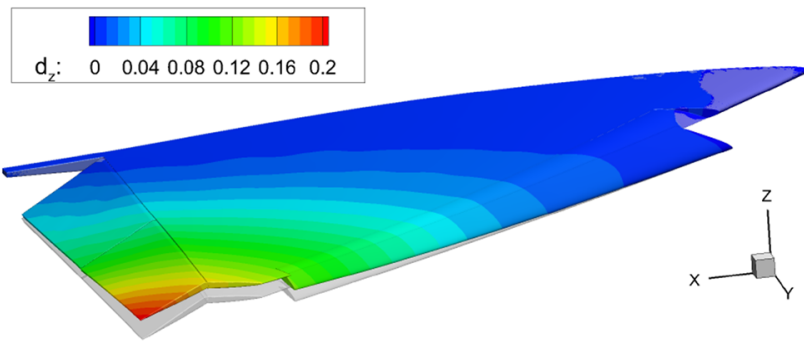

(d) $\alpha=15^{\circ}$ and $\delta=30^{\circ}$.

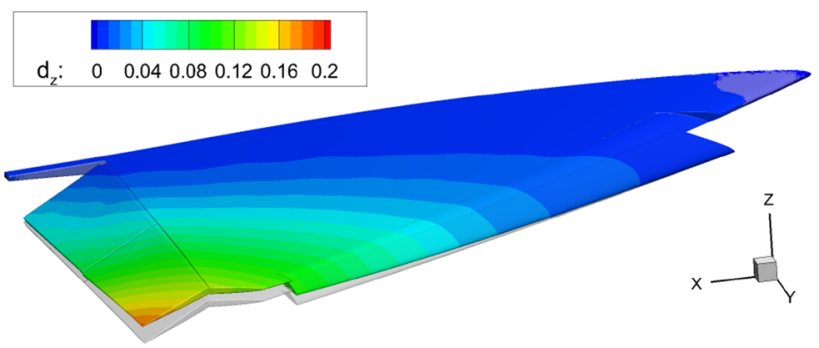

(f) $\alpha=20^{\circ}$ and $\delta=30^{\circ}$.

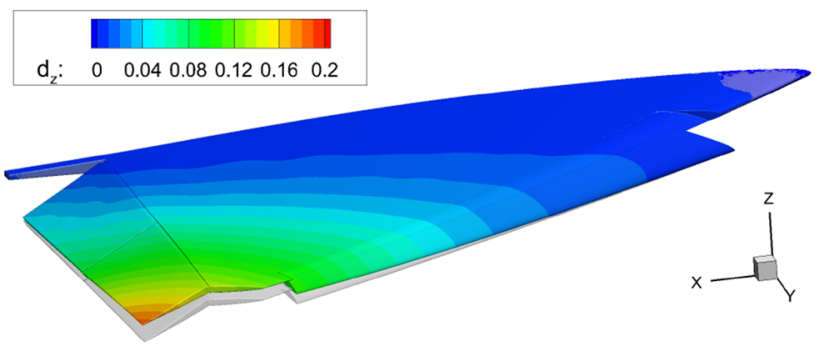

(h) $\alpha=25^{\circ}$ and $\delta=30^{\circ}$.

Fig. 11 Displacement in $z$-direction $d_{z}$ of the Model53 for $\mathrm{Ma}=0.8, \mathrm{Re}=15.1 \times 10^{7}, \alpha=\left[10^{\circ}, 15^{\circ}, 20^{\circ}, 25^{\circ}\right]$ and selected flap deflection angles $\delta$. The rigid wing is shown in translucent grey

flap deflection angle are similar for $\alpha=20^{\circ}$ and $\alpha=25^{\circ}$. For the pitching moment coefficient deviations such a clear trend could not be observed for downward flap deflections. This underlines the necessity of utilizing high-fidelity CFD-CSM simulations to capture the non-linear influence of flexibility on the vortical flow field. For downward flap deflections, the largest difference for lift has been observed at $\alpha=25^{\circ}$ and $\delta=20^{\circ}$ with $\Delta C_{\mathrm{L}}=-0.07$ and $\Delta C_{\mathrm{My}}=0.02$ compared to the rigid wing values $\left(C_{\mathrm{L} \text {,rigid }}=1.23\right.$ and $\left.C_{\mathrm{My} \text {,rigid }}=-0.125\right)$. These deviations result from increased wing twist and a change in flap deflection angle of $\Delta \delta=-3.2^{\circ}$. Compared to upward flap deflections, where the the deviations in lift and pitching moment could be mainly attributed to the change in effective flap deflection angle, wing twist plays a major 


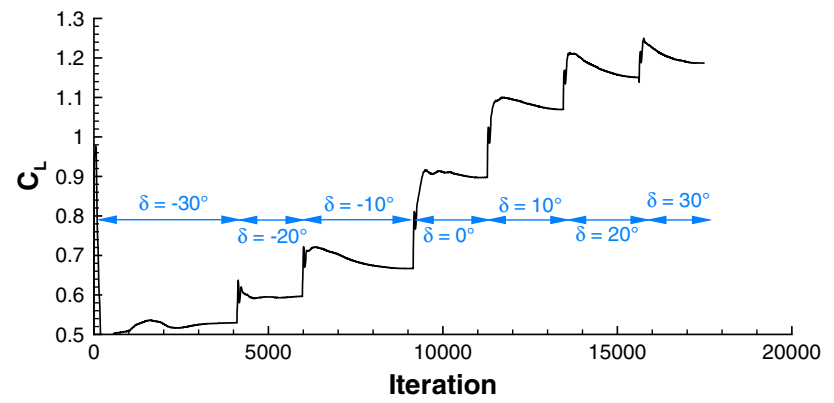

(a) Aerodynamic simulation.

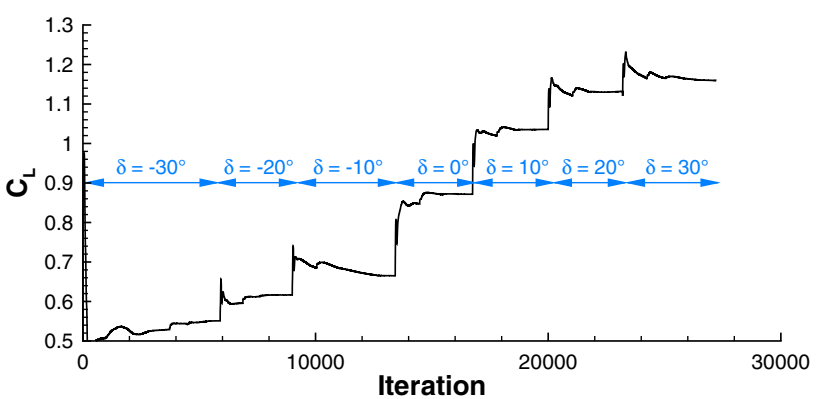

(b) Aeroelastic simulation.

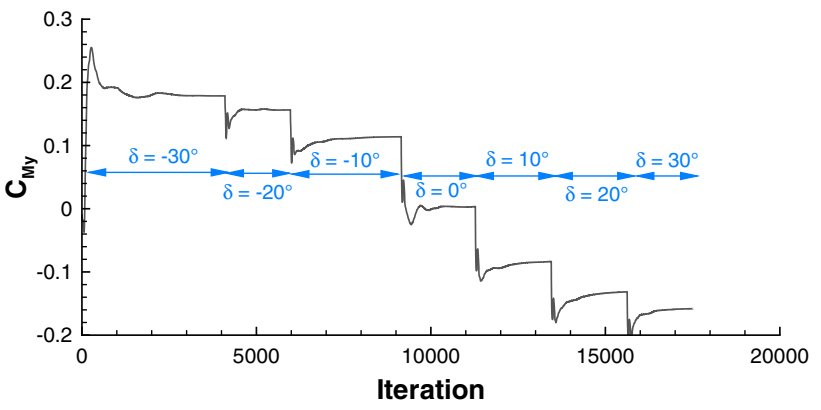

(c) Aerodynamic simulation.

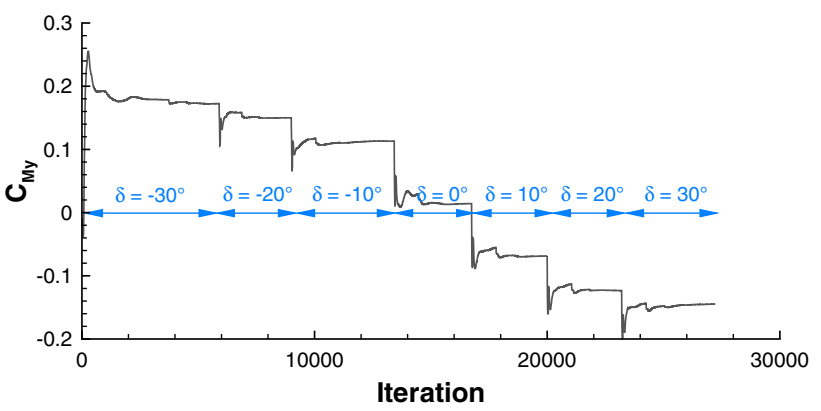

(d) Aeroelastic simulation.

Fig. 12 Convergence history of the lift and pitching moment coefficient for the aerodynamic and aeroelastic simulation at $\mathrm{Ma}=0.8$, $\alpha=20^{\circ}$

role additional to the change in effective deflection angle for downward flap deflections.

The analysis of the lift and pitching moment characteristics of the rigid and flexible configuration revealed the importance of taking the structural flexibility into account to obtain more accurate results for the considered range of flap deflections. Compared to the aerodynamic simulations, the computational time increased by a factor of 1.5 for the aeroelastic simulations. Thus, in case of high dynamic pressure, coupled CFD-CSM simulations are recommended for delta wings instead of CFD simulations to gain more accurate results.

Acknowledgements The fruitful collaboration with Airbus Defence and Space and its partial financial sponsoring is gratefully acknowledged. The authors also thank the Gauss Centre for Supercomputing e.V. (www.gauss-centre.eu) for supporting this project by providing computing time on the GCS Supercomputer SuperMUC-NG at Leibniz Supercomputing Centre (www.lrz.de).

Funding Open Access funding enabled and organized by Projekt DEAL.

Open Access This article is licensed under a Creative Commons Attribution 4.0 International License, which permits use, sharing, adaptation, distribution and reproduction in any medium or format, as long as you give appropriate credit to the original author(s) and the source, provide a link to the Creative Commons licence, and indicate if changes were made. The images or other third party material in this article are included in the article's Creative Commons licence, unless indicated otherwise in a credit line to the material. If material is not included in the article's Creative Commons licence and your intended use is not permitted by statutory regulation or exceeds the permitted use, you will need to obtain permission directly from the copyright holder. To view a copy of this licence, visit http://creativecommons.org/licenses/by/4.0/.

\section{References}

1. Breitsamter, C.: Aerodynamic efficiency of high maneuverable aircraft applying adaptive wing trailing edge section. In: 24th International Congress of the Aeronautical Sciences, pp. 1-10 (2004)

2. Hövelmann, A., Pfnür, S., Breitsamter, C.: Flap efficiency analysis for the SAGITTA diamond wing demonstrator configuration. CEAS Aeronaut. J. 6(4), 497-514 (2015)

3. Pfnür, S., Oppelt, S., Breitsamter, C.: Yaw-control efficiency analysis for a diamond wing configuration with outboard split flaps. CEAS Aeronaut. J. 10(2), 645-663 (2019)

4. Helm, S., Haupt, M., Stickan, B., Bleecke, H.: Advancements of CFD-CSM coupling by means of multibody simulation. In: Deutscher Luft- und Raumfahrtkongress. Deutsche Gesellschaft für Luft- und Raumfahrt-Lilienthal-Oberth e.V. (2015)

5. Capsada, L.A., Heinrich, R.: Development of the DLR TAU code for modelling of control surfaces. In: Deutscher Luft- und Raumfahrtkongress. Deutsche Gesellschaft für Luft- und RaumfahrtLilienthal-Oberth e.V. (2019). https://elib.dlr.de/126777/

6. Heinrich, R., Reimer, L., Michler, A., Ernst, B., Ritter, M., Neumann, J.: DLR pilot applications in ComFlite. In: Computational Flight Testing, pp. 235-247. Springer. Braunschweig, Germany. (2013)

7. Kroll, N., Abu-Zurayk, M., Dimitrov, D., Franz, T., Führer, T., Gerhold, T., Görtz, S., Heinrich, R., Ilic, C., Jepsen, J., et al.: DLR project Digital-X: towards virtual aircraft design and flight testing based on high-fidelity methods. CEAS Aeronaut. J. 7(1), $3-27$ (2016) 
8. Bramsiepe, K., Voß, A., Klimmek, T.: Design and sizing of an aeroelastic composite model for a flying wing configuration with maneuver, gust, and landing loads. CEAS Aeronaut. J. 11(3), 677-691 (2020)

9. Vos, J.B., Charbonnier, D., Ludwig, T., Merazzi, S., Gehri, A., Stephani, P.: Recent Developments on Fluid Structure Interaction Using the Navier Stokes Multi Block (NSMB) CFD Solver. https://doi.org/10.2514/6.2017-4458

10. Sørensen, K., Tremel, U., Rieger, H., Hitzel, S.: Simulation of Manoeuvring aircraft with the unstructured Chimera approach. In: 45th AIAA Aerospace Sciences Meeting and Exhibit. Reno, Nevada (2007). https://doi.org/10.2514/6.2007-123

11. Clifton, J., Ratcliff, C., Bodkin, D.: Determining the stability and control characteristics of high-performance maneuvering aircraft using high-resolution CFD simulation with and without moving control surfaces. https://doi.org/10.2514/6.2013-972

12. Voß, A.: Comparing VLM and CFD maneuver loads calculations for a flying wing configuration. In: IFASD 2019-International Forum on Aeroelasticity and Structural Dynamics (2019). https:// elib.dlr.de/127750/. Accessed 25 July 2019

13. Fornasier, L., Rieger, H., Tremel, U., van der Weide, E.: Timedependent aeroelastic simulation of rapid manoeuvring aircraft. https://doi.org/10.2514/6.2002-949

14. Stickan, B., Bleecke, H., Silvio, S., Radespiel, R., Burg, J.W., Sørensen, K.: NASTRAN Based Static CFD-CSM Coupling in FlowSimulator, pp. 223-234. Springer, Berlin (2013). https://doi. org/10.1007/978-3-642-38877-4_15

15. Tremel, U., Deister., F., Sørensen, K., Rieger, H., Weatherill, N.: The SimServer-a parallel multidisciplinary simulation environment. Konferenzband DGLR Jahrestagung Beitrag DGLR-2003088 Munich Germany 17, 20 (2003)

16. Kroll, N., Langer, S., Schwöppe, A.: The DLR flow solver TAUstatus and recent algorithmic developments. In: 52th AIAA Aerospace Sciences Meeting. Maryland, USA (2014)
17. Spalart, P., Allmaras, S.: A one-equation turbulence model for aerodynamic flows (1992). https://doi.org/10.2514/6.1992-439

18. Edwards, J., Chandra, S.: Comparison of eddy viscosity-transport turbulence models for three-dimensional, shock-separated flowfields (1994). https://doi.org/10.2514/6.1994-2275

19. Scott, R., Hoadley, S., Wieseman, C., Durham, M.: Benchmark active controls technology model aerodynamic data. J. Guidance Control Dyn. 23(5), 914-921 (2000)

20. Ballmann, J., Boucke, A., Chen, B., Reimer, L., Behr, M., Dafnis, A., Buxel, C., Buesing, S., et al.: Aero-structural wind tunnel experiments with elastic wing models at high Reynolds numbers (HIRENASD-ASDMAD). In: 49th AIAA Aerospace Sciences Meeting Including the New Horizons Forum and Aerospace Exposition, p. 882

21. Gerhold, T., Neumann, J.: The parallel mesh deformation of the DLR TAU-Code (2007). https://doi.org/10.1007/978-3-54074460-3_20

22. Burggraf, U., Kuntz, M., Schöning, B.: Implementation of the Chimera Method in the Unstructured DLR Finite Volume Code TAU, pp. 93-100. Vieweg+Teubner Verlag, Wiesbaden (1999). https://doi.org/10.1007/978-3-663-10901-3_13

23. Moioli, M., Reinbold, C., Sørensen, K., Breitsamter, C.: Investigation of additively manufactured wind tunnel models with integrated pressure taps for vortex flow analysis. Aerospace 6(10), 113 (2019)

24. Maierl, R., Petersson, O., Daoud, F., Bletzinger, K.: Automatically generated aeroelastic analysis models including physics based control surface representation. In: 4th Aircraft Structural Design Conference (2014)

Publisher's Note Springer Nature remains neutral with regard to jurisdictional claims in published maps and institutional affiliations. 